\title{
Inventaire spécifique et caractéristiques de quelques frayères du lac de Buyo dans le Parc National de Taï (Sud-Ouest Côte d'Ivoire)
}

\author{
N'DRI Olga Rosemonde ${ }^{1}$, KONAN Yao Aristide ${ }^{2}$, MONNEY Attoubé Ida ${ }^{1}$, KONE Tidiani1 \\ 1'Laboratoire de Biodiversité et Ecologie Tropicale, UFR Environnement, Université Jean Lorougnon Guédé, BP 150 \\ Daloa, Côte d'Ivoire \\ 2Laboratoire d'Hydrobiologie et Ecotoxicologie des Eaux, UFR Biosciences, Université Félix Houphouët-Boigny, 22 \\ BP 586 Abidjan 22, Côte d'Ivoire \\ *Auteur de correspondance : ariskoya@yahoo.fr \\ Original submitted in on $20^{\text {th }}$ December 2019. Published online at www.m.elewa.org/journals/ on $31^{\text {st }}$ January 2020 \\ https://doi.org/10.35759/JABs.v145.5
}

\section{RESUME}

Objectifs : Des investigations ont été menées dans 11 sites du lac de Buyo situés sur le cours du fleuve Sassandra dans le Parc National de Taï (Côte d'Ivoire) en vue de connaître les espèces de poisson qui utilisent ces zones comme sites de reproduction.

Méthodologie et Résultats : Dans chaque site d'échantillonnage, les variables environnementales ont été mesurées et les poissons ont été capturés à l'aide de filets maillants. Les stades de maturité sexuelle avancés ont été considérés comme critères de reconnaissance des sites de fraie. La richesse spécifique a été de 32 espèces, répartie en 13 familles et dominée par les Cichlidae (7 espèces, soit 22\%). Sur la base des stades sexuels de maturité, 14 espèces, en phase de reproduction, ont été capturées dans les 11 sites d'échantillonnage. Les résultats de l'analyse des sédiments ont indiqué une prédominance de sable $(>60 \%)$ dans les sites de reproduction et des valeurs optimales de température $\left(28,52^{\circ} \mathrm{C}\right)$, de $\mathrm{pH}(6,53)$, de conductivité $(36,50 \mu \mathrm{S} / \mathrm{cm})$ et d'oxygène dissous $(5,37 \mathrm{mg} / \mathrm{l})$.

Conclusion et application des résultats : La présente étude a montré que les sites échantillonnés dans le lac de Buyo situe à l'intérieur du le Parc National de Taï sont caractérisés par des sédiments sableux et constituent des frayères pour plusieurs espèces de poisson. La localisation de ces zones de reproduction représente donc un outil important pour les gestionnaires dans la conservation de la diversité piscicole du parc national de Taï.

Mots clés : sites de frai, stade de maturité sexuelle, ichtyofaune, lac de barrage de Buyo. 
Specific inventory and characteristics of some spawning grounds of Lake Buyo in the Taï National Park (South-West Côte d'Ivoire)

\section{Abstract}

Objectives: Investigations were carried out in 11 sites of Lake Buyo located in the midstream of Sassandra River in Taï National Park (Côte d'Ivoire) to get to know fish species that use these areas as spawning sites.

Methodology and Results: Environmental variables were measured in each sampling sites and fish were caught using gillnets. Advanced stages of sexual maturity were considered as recognition criteria of spawning areas. Specific richness was of 32 species, belonging in 13 families and dominated by Cichlidae (7 species, 22\%). Based on advanced sexual maturity stages, 14 species in spawning period, were captured at the 11 sampling sites. Results of sediment analysis indicated a predominance of sand $(>60 \%)$ in spawning sites and optimal values of temperature $\left(28.52^{\circ} \mathrm{C}\right), \mathrm{pH}(6,53)$, conductivity $(36,50 \mu \mathrm{S} / \mathrm{cm})$ and dissolved oxygen $(5,37 \mathrm{mg} / \mathrm{l})$.

Conclusion and application of results: The present study showed that sites sampled in Lake Buyo located within the Tai National Park are characterized by sandy sediments and constitute spawning areas for several fish species. The location of these spawning areas therefore is an important tool for managers in conserving fish diversity of Taï National Park.

Keywords: spawning sites, stage of sexual maturity, Ichtyofauna, Buyo Dam Lake.

\section{INTRODUCTION}

Les pressions sur les milieux naturels sont de plus en plus fréquentes ces dernières décennies ayant pour conséquences, entre autres, l'altération des écosystèmes aquatiques (Pimm et al., 1995; Sanderson et al., 2002). Ainsi, le maintien des services environnementaux ou écologiques demeure une préoccupation majeure se traduisant par le développement de méthodes de caractérisation de l'environnement, de diagnostic des milieux et de restauration des écosystèmes dégradés (Daily et al., 2000 ; Rosenzweig, 2003). Parmi ces milieux, les lacs abritent une importante biodiversité animale et sont sources de nombreux services écosystémiques (Ostendorp et al., 2004). En Côte d'Ivoire, le lac de Buyo subit l'influence d'importantes activités anthropiques dominées par l'agriculture et la pêche. En effet, les pressions de pêche pratiquées par les populations établies autour du lac, couplée à l'utilisation d'engins de capture non réglementaires ont pour conséquences la destruction des stocks et la disparition d'espèces de poissons. Or ce lac

\section{MATERIAL AND METHODES}

Le lac de Buyo est un lac artificiel situé sur le fleuve Sassandra au sud-ouest de la Côte d'lvoire. II est compris entre $06^{\circ} 54^{\prime}$ et $07^{\circ} 31^{\prime}$ de longitude ouest et possède une faune diversifiée avec quarante-cinq espèces de poissons (Goli Bi et al., 2019) et la partie du lac située à l'intérieur du Parc National de Taï (PNT) n'échappe pas à ces menaces en raison de la présence permanente de plusieurs campements de pêcheurs professionnels. Dans ces conditions, un aménagement des pêcheries dans cette zone est nécessaire et passe par l'identification des sites de frai, leur caractérisation et la connaissance de leur fonctionnement. Les études ichtyologiques menées dans le lac de Buyo se sont intéressées à la diversité piscicole, aux périodes de reproduction de plusieurs espèces de poissons et à l'écologie alimentaire (Kouamé et al., 2006 ; Kouassi et al., 2019) et n'ont pas permis de cerner les lieux de ponte des poissons. La présente étude se propose donc de faire l'inventaire des espèces de poissons des frayères et d'en connaitre les caractéristiques environnementales, biologiques et écologiques afin de les préserver.

$01^{\circ} 14^{\prime}$ et $07^{\circ} 03^{\prime}$ de latitude nord et couvre une superficie de $920 \mathrm{~km}^{2}$ avec un bassin versant de 75 $000 \mathrm{~km}^{2}$. Le régime hydrologique du lac dépend de 
celui du fleuve Sassandra, des affluents du N'Zo et des précipitations de la région (Traoré et Konan, 1989). La variation du niveau d'eau se matérialise par la mise en évidence d'une zone de marnage dont la surface varie en fonction des régimes hydrologiques et pluviométriques (Traoré, 1996). Au total, 11 sites d'échantillonnage ont été choisis dans la partie du lac située dans le Parc National de Taï en fonction de la diversité des habitats, du régime hydrologique et l'accessibilité (Figure 1)

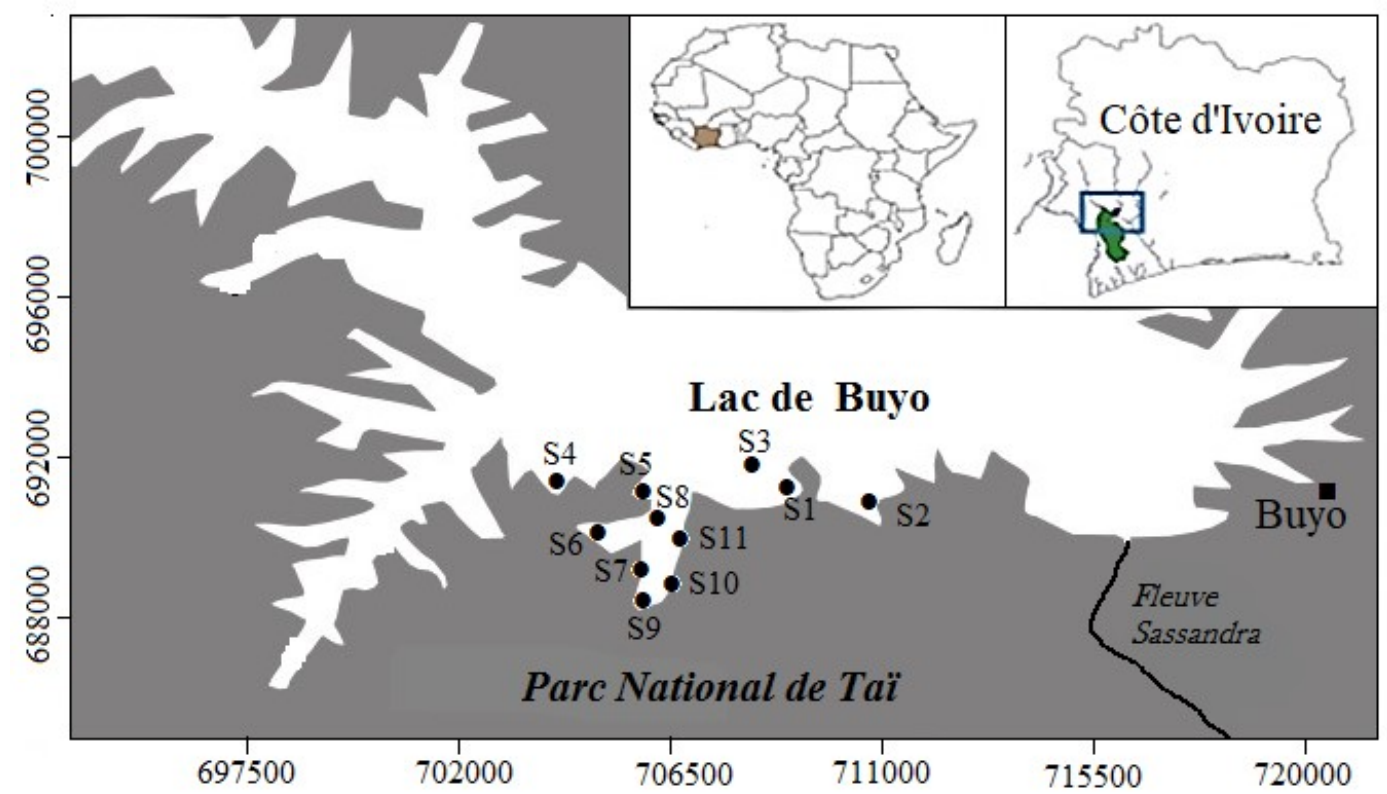

Figure 1. Localisation des sites d'échantillonnage des poissons dans le lac de Buyo (Côte d'Ivoire).

Collecte des données: Les mesures des variables environnementales ont été effectuées in situ mensuellement entre juin 2018 et mai 2019. Le taux d'oxygène dissous, le $\mathrm{pH}$, la conductivité et la température de l'eau ont été mesurés à l'aide d'un mulitiparamètre de type Waterproof DO 330. La transparence et la profondeur ont été mesurées à l'aide d'un disque de Secchi et d'un bâton gradué et lesté, respectivement. Dans chaque site, deux mesures ont été effectuées et la moyenne des mesures est rapportée à la zone en question. Des échantillons de sédiments ont également été prélevés, séchés et tamisés afin d'évaluer la distribution granulométrique (Bruton et Gophen, 1992). L'échantillonnage des poissons a été réalisé à l'aide d'une batterie de 10 filets monofilaments de vide de maille $6,8,10,15,18,20$, $25,30,35$ et $40 \mathrm{~mm}$ de $30 \mathrm{~m}$ de longueur pour une hauteur de chute de 1,5 m. D'autres engins tels que des nasses et des éperviers ont été utilisés et les poissons capturés ont été identifiés selon Paugy et al (2003 a et b) puis dénombrés. Les gonades de chaque spécimen ont été prélevées après dissection puis le sexe et les stades de maturité sexuelle ont été déterminés selon Fontana (1969) et N'Goran (1995)
(Tableau 1). Les individus possédant des gonades aux stades de maturités sexuelles I, II et III ont été considérés comme immatures tandis que ceux qui avaient des gonades aux stades de maturité sexuelle $I V$, $V$ et $\mathrm{VI}$ ont été pris pour des individus matures en phase de reproduction (Heyman et Kjerfve, 2008). Un site d'échantillonnage a été considéré comme site de frai pour une espèce donnée lorsque: $1 /$ le nombre d'individus capturés dans ce site est supérieur ou égal à 10 et 2/ l'abondance des géniteurs aux stades de maturités sexuelles IV, $\mathrm{V}$ et $\mathrm{VI}$ est supérieure ou égale à $70 \%$ (Heyman et Kjerfve, 2008).

Analyses statistiques : Les données de granulométrie ont été soumises à une analyse de classification hiérarchique ascendante, afin de pouvoir regrouper les sites d'échantillonnage dont les caractéristiques sont plus proches. Les paramètres physico-chimiques ont été comparés en fonction des sites d'échantillonnage par le test de Kruskall-Wallis et un dendrogramme a été réalisé sur la base des variables environnementales et de la granulométrie des sédiments afin d'analyser les similarités entre ces sites. Les analyses ont été réalisées à l'aide du logiciel Statistica 7.1. 
Tableau 1. Echelle macroscopique d'identification des stades de maturation sexuelle d'après Fontana (1969) et N'Goran (1995).

\begin{tabular}{|c|c|c|}
\hline Stades & Femelle & Mâle \\
\hline 1 & $\begin{array}{l}\text { Immature : Ovaire petit, ferme, de couleur rose clair ou } \\
\text { transparent. Ovocytes invisibles. }\end{array}$ & $\begin{array}{l}\text { Immature : Testicule blanc, légèrement } \\
\text { translucide, fin et en lame de couteau. }\end{array}$ \\
\hline 2 & $\begin{array}{l}\text { Début de maturation : Ovaire peu développé, de } \\
\text { volume plus important que le stade } 1 \text { et de couleur } \\
\text { rose. }\end{array}$ & $\begin{array}{l}\text { Début de maturation : Sensiblement identique } \\
\text { au stade 1, mais plus volumineux. }\end{array}$ \\
\hline 3 & $\begin{array}{l}\text { Maturation : Ovaire développé avec une couleur } \\
\text { variant du rose à l'orange clair. Les ovocytes sont peu } \\
\text { visibles à travers la membrane ovarienne. }\end{array}$ & $\begin{array}{l}\text { Maturation : Testicule ferme et blanchâtre. } \\
\text { Aucun liquide ne s'écoule à l'incision de la } \\
\text { gonade. }\end{array}$ \\
\hline 4 & $\begin{array}{l}\text { Pré-ponte : Gonade plus grosse et moins ferme, de } \\
\text { couleur orange clair à foncé. La surface de l'ovaire est } \\
\text { granuleuse du fait des ovocytes visibles à travers la } \\
\text { membrane ovarienne. }\end{array}$ & $\begin{array}{l}\text { Pré-émission : Testicule blanc et plus mou. Un } \\
\text { liquide blanchâtre s'écoule lorsqu'on effectue } \\
\text { une incision dans la gonade. }\end{array}$ \\
\hline 5 & $\begin{array}{l}\text { Ponte : L'ovaire est très gros, de couleur orange foncé } \\
\text { et occupe presque la totalité de la cavité abdominale. } \\
\text { La membrane ovarienne est très fine et les ovocytes } \\
\text { sont expulsés à la moindre pression abdominale. }\end{array}$ & $\begin{array}{l}\text { Emission : Gonade grosse et molle. Le sperme } \\
\text { coule à la moindre pression abdominale. }\end{array}$ \\
\hline 6 & $\begin{array}{l}\text { Post-ponte : L'ovaire est flasque, très vascularisé et de } \\
\text { couleur variant du rose saumon au rouge. Des } \\
\text { ovocytes plus petits et des espaces hyalins sont } \\
\text { visibles à travers la membrane ovarienne. }\end{array}$ & $\begin{array}{l}\text { Post-émission : Gonade flasque présentant } \\
\text { une vascularisation très fine surtout dans la } \\
\text { partie postérieure. }\end{array}$ \\
\hline
\end{tabular}

\section{RÉSULTATS}

Variables environnementales : Le Tableau 2 présente les variations des paramètres physico-chimiques entre les sites d'échantillonnage (de S1 à S11). Les variations de température sont faibles et comprises entre $27,91^{\circ} \mathrm{C}$ et $29,66^{\circ} \mathrm{C}$. Le taux d'oxygène dissous varie entre $4,35 \mathrm{mg} / \mathrm{l}$ et $6,54 \mathrm{mg} / \mathrm{l}$ et les eaux sont généralement plus oxygénées dans les sites $\mathrm{S} 3, \mathrm{~S} 8$ et S11 (5,75-6,54mg/l). La conductivité moyenne oscille entre 28,75 et $43,83 \mu \mathrm{s} / \mathrm{cm}$ et les valeurs les plus élevées ont été obtenues durant les saisons pluvieuses dans la majorité des sites d'échantillonnage (Tableau 2). Les eaux des différents sites sont relativement acides avec des valeurs moyennes de $\mathrm{pH}$ oscillant entre 6,16 et 6,68. Les valeurs les plus élevées de la profondeur ont été enregistrées à S3 $(10,61 \mathrm{~m})$ et S8 $(7,35 \mathrm{~m})$ tandis que la transparence la plus importante a été obtenue à $\mathrm{S} 1$ et $\mathrm{S} 3(1,20 \mathrm{~m})$. La comparaison de ces paramètres entre les sites a présenté des différences significatives (Kruskall-Wallis; $p<0,05$ ). Concernant la variation temporelle, seule la comparaison de la transparence et la profondeur ont indiqué des différences significatives entre les saisons sèche et pluvieuse (Kruskall-Wallis ; $p<0,05$ ). L'analyse du sédiment a montré qu'il est composé de gravier, de sable et limon et d'argile (Figure 2). Les proportions de sable sont plus élevées dans tous les sites, variant entre $62,16 \%$ et $82,84 \%$ à l'exception du site S4 où la valeur est inférieure à $50 \%$ (41,39\%). Les proportions de gravier sont faibles aux sites S3 $(3,26 \%)$ et S8 $(0,69 \%)$ et élevés à $S 4(54,55 \%)$. Concernant le limon et l'argile, les valeurs sont faibles dans tous les sites $(<5 \%)$. Le dendrogramme obtenu (Figure 3 ) a permis de regrouper les sites d'échantillonnage en trois grands groupes:

- le groupe 1 qui comprend le site S4 dont le sédiment est dominé par le gravier,

- $\quad$ le groupe 2 constitué par les sites S3 à S8 caractérisés par une teneur moyenne de gravier, - $\quad$ le groupe 3 composé par les sites S1, S2, S5 à $\mathrm{S} 7$ et $\mathrm{S} 9$ à $\mathrm{S} 11$ qui se distinguent par leur sédiment dominé par le sable. 


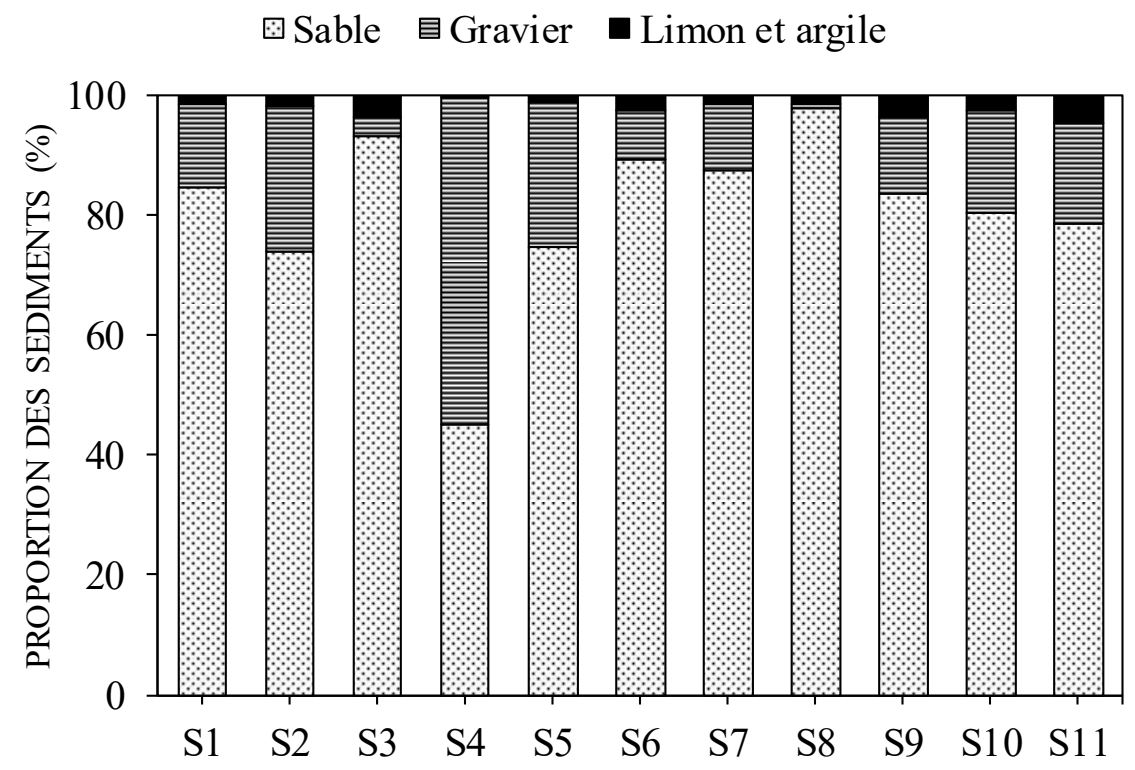

Figure 2. Composition du substrat (en pourcentage) des 11 sites échantillonnés dans le lac de Buyo (Côte d'Ivoire) de juin 2018 à mai 2019.

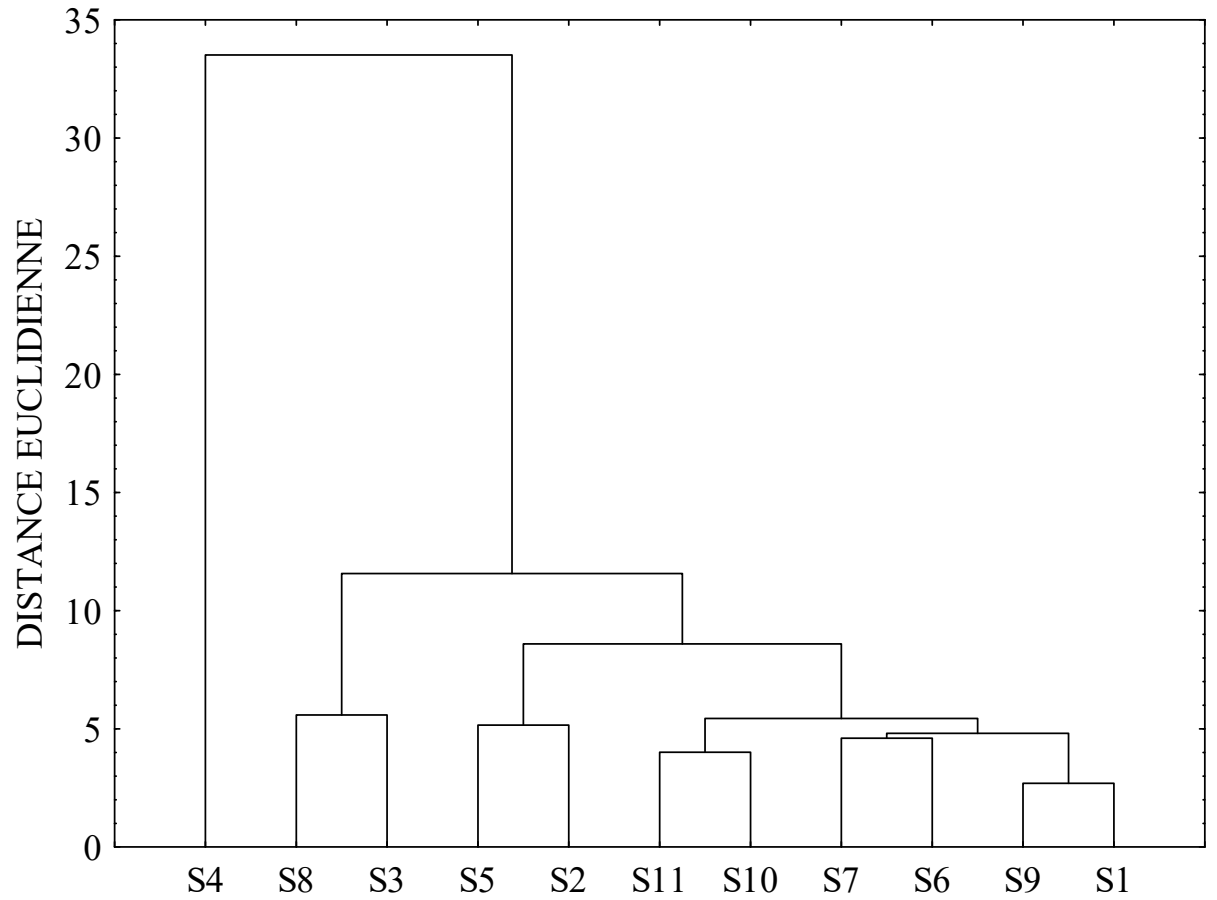

Figure 3. Dendrogramme montrant la similarité des sites d'échantillonnage du lac de Buyo (Côte d'Ivoire).

Richesse spécifique: Les captures de poissons se répartissent en 32 espèces et 13 familles (Tableau 3). Les Cichlidae dominent le nombre d'espèces capturées (7 espèces soit 22\%), suivie les Cyprinidae (5 espèces, $16 \%$ ) et les Mormyridae (4 espèces, $13 \%$ ) tandis que les Clupeidae, Osteoglossidae, Distichodontidae,
Malapteruridae et Centropomidae sont monospécifiques.

Variation spatiale et temporelle du peuplement : Sur les 32 espèces récoltées, les sites $\mathrm{S} 1$ et $\mathrm{S} 6$ ont présenté une richesse spécifique plus élevée (20 et 22 espèces, respectivement) tandis que les sites $\mathrm{S} 7$ et $\mathrm{S} 8$ ont été moins diversifiées (11 et 12 espèces, 
respectivement). Par ailleurs, les sites $\mathrm{S} 6$ et $\mathrm{S} 11$ ont compté le plus grand nombre d'individus $(21,58 \%$ et $16,26 \%$ de l'abondance totale, respectivement) alors que $\mathrm{S} 7$ et $\mathrm{S} 8$, des effectifs plus faibles $(4,60 \%$ et $2,35 \%$, respectivement). Les espèces qui présentent le plus grand nombre d'individus dans tous les sites échantillonnés ( $>5 \%$ de l'effectif total) sont Coptodon zillii $(26,5 \%)$, Barbus macrops (13\%), Oreochromis niloticus $(8,3 \%)$, Chrysichthys nigrodigitatus $(7,7 \%)$, Synodontis punctifer $(7,1 \%)$, Schilbe mandibularis $(6,4 \%)$ et Hemichromis fasciatus $(5,4 \%)$. Parmi ces espèces, seules $B$. macrops, $C$. nigrodigitatus et Lates niloticus ont été capturées dans tous les sites. La comparaison de la richesse spécifique en fonction des sites a montré des différences significatives (KruskallWallis, $p<0,05)$. Dans tous les sites échantillonnés, la richesse spécifique a été plus élevée en saison des pluies qu'en saison sèche et les différences ont été significatives (Kruskall-Wallis, $p<0,05$ ).

Occurrence des individus matures et immatures dans les sites d'échantillonnage: Des individus matures (stades de maturité $\mathrm{IV}, \mathrm{V}$ et $\mathrm{VI}$ ) ont été observées chez toutes les espèces récoltées. Toutefois, seules 14 espèces de poisson ont présenté des proportions des gonades matures supérieures ou égales à $70 \%$ de l'effectif total (Tableau 4). Parmi ces espèces qui viennent faire leur fraie dans des certains la zone d'étude, Barbus macrops et Coptodon zillii ont été recensées dans la majorité des sites d'échantillonnage (7 et 8 sites, respectivement) tandis que Schilbe mandibularis a été capturée dans 4 sites (Tableau 4). Quant aux espèces Chrysichthys maurus, Malapterurus electricus, Synodontis punctifer et Hemichromis bimaculatus, ils font leur fraie sur un seul site (S4, S6, S3 et S6, respectivement). Les individus immatures ont été observés dans tous les sites d'échantillonnage avec des proportions variables selon les espèces. Chez les 4 espèces Pellonula leonensis, Mormyrus rume, Lates niloticus et Sarotherodon melanotheron, les individus immatures ont été plus abondants que les matures (Tableau 4). En effet, les immatures ont été uniquement récoltés $(100 \%)$ dans les sites S5 et S6 pour $P$. leonensis, S6 pour M. rume, S8 pour $C$. nigrodigitatus, $\mathrm{S} 6$ pour $C$. maurus, $S 4$ pour S. punctifer, S1 et S4 pour O. niloticus. Les périodes de capture des géniteurs aux stades de maturité sexuelle IV, V et VI s'étendent, globalement, de mai à juillet (Tableau 5). Barbus macrops a été capturée durant 9 campagnes d'échantillonnage (de janvier à septembre) tandis que Chrysichthys nigrodigitatus, Coptodon zillii et Oreochromis niloticus ont été capturés pendant 8 sorties de terrain (sur 12 sorties au total). Par contre, les individus matures de Chrysichthys maurus et Malapterurus electricus ont été capturés une seule fois en juillet aux sites S4 et S6, respectivement.

Tableau 2. Valeurs moyennes \pm écart-type des paramètres physicochimiques mesurés dans 11 sites d'échantillonnage (S1 à S11) du lac de Buyo (Côte d'Ivoire) entre juin 2018 et mai 2019.

\begin{tabular}{cccccccc}
\hline Sites & Saisons & Temp $\left.^{\circ} \mathbf{C}\right)$ & OD $(\mathbf{m g} / \mathbf{l})$ & Cond $(\boldsymbol{\mu s} / \mathbf{c m})$ & pH & Prof $(\mathbf{m})$ & Transp (m) \\
\hline S1 & SS & $27,92 \pm 1,01$ & $5,02 \pm 0,96$ & $33,00 \pm 4,69$ & $6,80 \pm 0,63$ & $5,54 \pm 2,42$ & $1,20 \pm 0,28$ \\
& SP & $28,75 \pm 1,23$ & $5,17 \pm 0,55$ & $41,08 \pm 10,71$ & $6,59 \pm 0,89$ & $2,59 \pm 1,01$ & $0,82 \pm 0,26$ \\
\hline S2 & SS & $28,21 \pm 1,19$ & $5,47 \pm 0,79$ & $32,25 \pm 8,66$ & $6,62 \pm 0,23$ & $4,96 \pm 1,36$ & $1,17 \pm 0,22$ \\
& SP & $29,12 \pm 1,47$ & $5,06 \pm 0,83$ & $40,02 \pm 7,61$ & $6,46 \pm 0,90$ & $2,40 \pm 1,16$ & $0,87 \pm 0,28$ \\
\hline S3 & SS & $28,05 \pm 1,09$ & $6,20 \pm 0,46$ & $33,75 \pm 4,65$ & $6,40 \pm 0,52$ & $10,61 \pm 1,30$ & $1,20 \pm 0,41$ \\
& SP & $28,82 \pm 1,27$ & $5,35 \pm 1,01$ & $38,08 \pm 9,28$ & $6,68 \pm 0,73$ & $6,22 \pm 2,28$ & $1,06 \pm 0,11$ \\
\hline S4 & SS & $28,32 \pm 1,64$ & $6,06 \pm 0,83$ & $29,00 \pm 9,76$ & $6,66 \pm 0,74$ & $5,65 \pm 3,23$ & $1,08 \pm 0,12$ \\
& SP & $28,46 \pm 1,30$ & $5,00 \pm 1,55$ & $40,28 \pm 7,14$ & $6,61 \pm 0,96$ & $3,12 \pm 1,61$ & $0,93 \pm 0,24$ \\
\hline S5 & SS & $28,13 \pm 1,36$ & $5,98 \pm 0,92$ & $28,75 \pm 8,65$ & $6,83 \pm 0,43$ & $7,33 \pm 2,06$ & $1,10 \pm 0,33$ \\
& SP & $28,48 \pm 1,54$ & $4,55 \pm 0,65$ & $39,24 \pm 8,45$ & $6,41 \pm 0,55$ & $3,09 \pm 2,20$ & $0,68 \pm 0,21$ \\
\hline S6 & SS & $28,43 \pm 1,45$ & $5,85 \pm 1,12$ & $31,25 \pm 12,81$ & $6,69 \pm 0,65$ & $4,81 \pm 0,87$ & $1,13 \pm 0,29$ \\
& SP & $28,33 \pm 1,58$ & $5,59 \pm 0,74$ & $34,00 \pm 9,59$ & $6,86 \pm 0,97$ & $1,60 \pm 1,46$ & $0,52 \pm 0,20$ \\
\hline S7 & SS & $28,20 \pm 1,56$ & $4,35 \pm 2,40$ & $29,75 \pm 12,68$ & $6,63 \pm 0,67$ & $3,66 \pm 0,18$ & $1,00 \pm 0,31$ \\
& SP & $29,59 \pm 0,47$ & $5,74 \pm 0,40$ & $39,57 \pm 3,61$ & $6,12 \pm 0,46$ & $1,66 \pm 0,84$ & $0,70 \pm 0,16$ \\
\hline S8 & SS & $28,57 \pm 1,65$ & $6,17 \pm 0,38$ & $41,00 \pm 27,76$ & $6,49 \pm 0,46$ & $7,35 \pm 1,13$ & $1,02 \pm 0,18$ \\
& SP & $28,28 \pm 1,59$ & $5,84 \pm 0,90$ & $38,72 \pm 8,71$ & $6,78 \pm 0,73$ & $3,83 \pm 3,15$ & $0,64 \pm 0,29$ \\
\hline S9 & SS & $28,48 \pm 1,60$ & $4,47 \pm 0,53$ & $32,00 \pm 13,29$ & $6,31 \pm 0,39$ & $3,68 \pm 1,21$ & $0,83 \pm 0,08$
\end{tabular}


N'Dri et al., J. Appl. Biosci. 2020 Inventaire spécifique et caractéristiques de quelques frayères du lac de Buyo dans le Parc National de Taï (Sud-Ouest Côte d'Ivoire)

\begin{tabular}{cccccccc} 
& SP & $28,17 \pm 2,76$ & $5,01 \pm 0,41$ & $43,83 \pm 8,75$ & $5,96 \pm 0,62$ & $1,82 \pm 1,94$ & $0,28 \pm 0,21$ \\
\hline S10 & SS & $28,35 \pm 1,48$ & $4,73 \pm 0,87$ & $35,50 \pm 20,34$ & $6,24 \pm 0,34$ & $3,39 \pm 1,91$ & $1,08 \pm 0,53$ \\
& SP & $29,66 \pm 0,11$ & $5,66 \pm 1,56$ & $41,90 \pm 4,26$ & $7,01 \pm 0,54$ & $1,24 \pm 0,78$ & $0,54 \pm 0,45$ \\
\hline S11 & SS & $28,25 \pm 1,21$ & $5,04 \pm 0,60$ & $37,25 \pm 15,17$ & $6,18 \pm 0,34$ & $4,09 \pm 1,45$ & $1,14 \pm 0,28$ \\
& SP & $28,74 \pm 1,75$ & $6,54 \pm 1,21$ & $41,32 \pm 6,03$ & $6,44 \pm 0,69$ & $2,99 \pm 2,85$ & $0,62 \pm 0,28$ \\
\hline
\end{tabular}

Temp = Température ; OD= Oxygène dissous $;$ Cond $=$ Conductivité $;$ Prof $=$ Profondeur $;$ Transp = Transparence.

Tableau 3. Richesse spécifique, composition et distribution des poissons échantillonnés dans 11 sites d'échantillonnage u lac du Buyo (Côte d'Ivoire) de juin 2018 à mai 2019.

\begin{tabular}{|c|c|c|c|c|c|c|c|c|c|c|c|c|}
\hline Familles & Espèces & S1 & S2 & S3 & S4 & S5 & S6 & S7 & S8 & S9 & S10 & S11 \\
\hline Clupeidae & Pellonula leonensis & + & & + & + & + & + & + & + & + & & + \\
\hline Osteoglossidae & Heterotis niloticus & & & & & & & + & & + & & \\
\hline Mormyridae & $\begin{array}{l}\text { Mormyrus rume } \\
\text { Mormyrus ussheri } \\
\text { Mormyrus senegalensis } \\
\text { Mormyrops anguilloides }\end{array}$ & + & $\begin{array}{l}+ \\
+\end{array}$ & + & & + & $\begin{array}{l}+ \\
+\end{array}$ & & & & & + \\
\hline Alestidae & $\begin{array}{l}\text { Brycinus longipinnis } \\
\text { Brycinus imberi }\end{array}$ & $\begin{array}{l}+ \\
+\end{array}$ & + & $\begin{array}{l}+ \\
+ \\
\end{array}$ & + & $\begin{array}{l}+ \\
+\end{array}$ & + & & + & & $\begin{array}{l}+ \\
+\end{array}$ & $\begin{array}{l}+ \\
+ \\
\end{array}$ \\
\hline Distichodontidae & Distichodus rostratus & & & & & & + & & & & & \\
\hline Cyprinidae & $\begin{array}{l}\text { Barbus macrops } \\
\text { Barbus ablabes } \\
\text { Labeo coubie } \\
\text { Labeo parvus } \\
\text { Labeo senegalensis }\end{array}$ & $\begin{array}{l}+ \\
+\end{array}$ & $\begin{array}{l}+ \\
+\end{array}$ & $\begin{array}{l}+ \\
+\end{array}$ & $\begin{array}{l}+ \\
+\end{array}$ & + & $\begin{array}{l}+ \\
+ \\
+\end{array}$ & + & + & + & + & + \\
\hline Claroteidae & $\begin{array}{l}\text { Chrysichthys nigrodigitatus } \\
\text { Chrysichthys maurus }\end{array}$ & + & $\begin{array}{l}+ \\
+ \\
\end{array}$ & + & $\begin{array}{l}+ \\
+ \\
+\end{array}$ & $\begin{array}{l}+ \\
+ \\
\end{array}$ & $\begin{array}{l}+ \\
+ \\
\end{array}$ & + & + & + & + & + \\
\hline Schilbeidae & $\begin{array}{l}\text { Schilbe mandibularis } \\
\text { Schilbe intermedius }\end{array}$ & + & + & & + & + & $\begin{array}{l}+ \\
+\end{array}$ & + & + & + & + & + \\
\hline Clariidae & $\begin{array}{l}\text { Clarias anguillaris } \\
\text { Heterobranchus longifilis }\end{array}$ & + & + & & + & & + & + & & $\begin{array}{l}+ \\
+ \\
\end{array}$ & + & + \\
\hline Malapteruridae & Malapterurus electricus & + & & & & & + & & + & + & & + \\
\hline Mochokidae & $\begin{array}{l}\text { Synodontis punctifer } \\
\text { Synodontis koensis } \\
\text { Synodontis bastiani }\end{array}$ & $\begin{array}{l}+ \\
+ \\
+\end{array}$ & $\begin{array}{l}+ \\
+ \\
+\end{array}$ & $\begin{array}{l}+ \\
+\end{array}$ & $\begin{array}{l}+ \\
+\end{array}$ & $\begin{array}{l}+ \\
+\end{array}$ & + & & + & & $\begin{array}{l}+ \\
+\end{array}$ & + \\
\hline Centropomidae & Lates niloticus & + & + & + & + & + & + & + & + & + & + & + \\
\hline Cichlidae & $\begin{array}{l}\text { Coptodon zillii } \\
\text { Oreochromis niloticus } \\
\text { Hemichromis fasciatus } \\
\text { Hemichromis bimaculatus } \\
\text { Sarotherodon melanotheron } \\
\text { Sarotherodon galilaeus } \\
\text { Coptodon guineensis } \\
\end{array}$ & $\begin{array}{l}+ \\
+ \\
+ \\
+ \\
+ \\
+\end{array}$ & $\begin{array}{l}+ \\
+ \\
+ \\
+ \\
+ \\
+\end{array}$ & $\begin{array}{l}+ \\
+\end{array}$ & $\begin{array}{l}+ \\
+ \\
+\end{array}$ & $\begin{array}{l}+ \\
+ \\
+ \\
+ \\
+ \\
+\end{array}$ & $\begin{array}{l}+ \\
+ \\
+ \\
+ \\
+ \\
+\end{array}$ & $\begin{array}{l}+ \\
+ \\
+ \\
+ \\
+\end{array}$ & $\begin{array}{ll}+ \\
+\end{array}$ & $\begin{array}{l}+ \\
+ \\
+ \\
+ \\
+\end{array}$ & $\begin{array}{l}+ \\
+ \\
+ \\
+ \\
+ \\
+\end{array}$ & $\begin{array}{l}+ \\
+ \\
+ \\
+ \\
+\end{array}$ \\
\hline 13 & 32 & 20 & 19 & 12 & 15 & 17 & 22 & 12 & 11 & 15 & 15 & 17 \\
\hline
\end{tabular}


Tableau 4. Proportions des individus immatures (stades I, II et III) et matures (stades IV, V et VI) échantillonnés dans les 11 sites d'échantillonnage du lac de Buyo (Côte d'lvoire) de juin 2018 à mai 2019.

\begin{tabular}{|c|c|c|c|c|c|c|c|c|c|c|c|c|c|c|c|c|c|c|c|c|c|c|}
\hline Espèces $(\mathrm{N} \geq 10)$ & $\begin{array}{l}\text { S1 } \\
\text { Mat }\end{array}$ & $\mathrm{Imm}$ & $\begin{array}{l}\text { S2 } \\
\text { Mat }\end{array}$ & $\mathrm{Imm}$ & $\begin{array}{l}\text { S3 } \\
\text { Mat }\end{array}$ & $\mathrm{Imm}$ & $\begin{array}{l}\text { S4 } \\
\text { Mat }\end{array}$ & $\mathrm{Imm}$ & $\begin{array}{l}\text { S5 } \\
\text { Mat }\end{array}$ & $\mathrm{Imm}$ & $\begin{array}{l}\text { S6 } \\
\text { Mat }\end{array}$ & $\mathrm{Imm}$ & $\begin{array}{l}\text { S7 } \\
\text { Mat }\end{array}$ & $\mathrm{Imm}$ & $\begin{array}{l}\text { S8 } \\
\text { Mat }\end{array}$ & $\mathrm{Imm}$ & $\begin{array}{l}\text { S9 } \\
\text { Mat }\end{array}$ & Imm & $\begin{array}{l}\text { S10 } \\
\text { Mat }\end{array}$ & Imm & $\begin{array}{l}\text { S11 } \\
\text { Mat }\end{array}$ & $\mathrm{Imm}$ \\
\hline $\begin{array}{l}\text { P. leonensis } \\
\text { H. niloticus* }\end{array}$ & 19,1 & 81 & & & & & & & 0 & 100 & 0 & 100 & 81,8 & 18,2 & & & 84 & 24 & & & & \\
\hline
\end{tabular}

\section{M. rume}

$35,7 \quad 64,3$

$\begin{array}{lllll}\text { B. longipinnis* } & 100 & 0 & 77,8 & 22,2\end{array}$

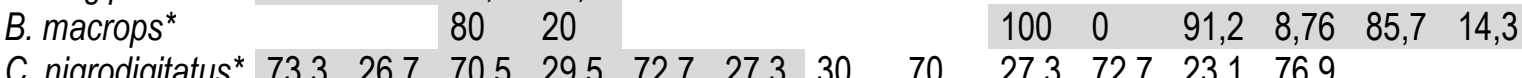

$0 \quad 100$

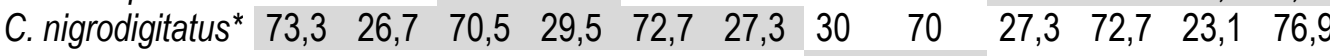

C. maurus* $\begin{array}{ll}76,9 & 23,1\end{array}$

S. mandibularis* $\quad 72,2 \quad 27,8 \quad 71,1 \quad 28,9$

$\begin{array}{llll}46,2 & 53,9 & 70,7 & 29,3\end{array}$

C. anguillaris*

M. electricus*

$83,8 \quad 16,2$

46,2 53,9

,

S. punctifer $46,2 \quad 53,9$ $\begin{array}{llllll}0 & 100 & 73,3 & 26,7 & 0 & 100\end{array}$ $\begin{array}{llllll}72,7 & 27,3 & 75 & 25 & 71,4 & 28,6\end{array}$

L. niloticus

$$
35,1 \quad 64,9
$$

C.zillii*

$$
\begin{array}{llll}
53,5 & 46,6 & 82,4 & 17,7
\end{array}
$$

O. niloticus*

$$
\begin{array}{llll}
53,5 & 46,6 & 82,4 & 17,7 \\
0 & 100 & 73,7 & 26,3
\end{array}
$$

H. fasciatus*

H. bimaculatus*

$0 \quad 100$

$$
33 \text {, }
$$

$\begin{array}{ll}83,3 & 16,7 \\ 44 & 56\end{array}$

$\begin{array}{llll}9,09 & 90,9 & 19,1 & 81\end{array}$

$\begin{array}{llll}23,5 & 76,5 & 28,6 & 71,4\end{array}$

$\begin{array}{llllllll}33,3 & 66,7 & 78,3 & 21,7 & 86,8 & 13,2 & 73,7 & 26,3\end{array}$

$\begin{array}{lllllllll}33,3 & 66,7 & 78,3 & 21,7 & 86,8 & 13,2 & 73,7 & 26,3 \\ 0 & 100 & 16,7 & 83,3 & 73,7 & 26,3 & 81,8 & 18,2\end{array}$

$\begin{array}{llll}72,7 & 27,3 & 87,1 & 12,9 \\ & & 75,5 & 24,5\end{array}$
0
$0 \quad 100$
$\begin{array}{llll} & 70 & 30\end{array}$
$\begin{array}{llllll}100 & 0 & 96,2 & 3,85 & 100 & 0 \\ 5 & 75 & 25,9 & 74,1\end{array}$

S. melanotheron

$33,3 \quad 66,7$

4060

$72 \quad 28$

1000

$\mathrm{N}=$ effectif total des individus d'une espèce dans chaque site, ${ }^{*}=$ espèces ayant des pourcentages d'individus matures $\geq 70 \%$. 
Tableau 5 : Périodes de capture des espèces de poissons dont les proportions des gonades matures (stades IV, V et $\mathrm{VI}$ ) sont supérieures ou égales à $70 \%(\mathrm{~N} \geq 10)$.

\begin{tabular}{lcl}
\hline Espèces & Nombre de sites de frai & Période de capture \\
\hline H. niloticus & 2 & septembre-octobre \\
B.longipinnis & 3 & juin-juillet \\
B.macrops & 7 & janvier à septembre \\
C.nigrodigitatus & 3 & février à septembre \\
C. maurus & 1 & juillet \\
S. mandibularis & 4 & mai à août \\
C. anguillaris & 2 & juin-juillet \\
M. electricus & 1 & juillet \\
S. punctifer & 1 & mai à septembre \\
S. koensis & 3 & juillet à octobre \\
C.zillii & 8 & janvier à août \\
O. niloticus & 3 & janvier à avril ; juillet à octobre \\
H. fasciatus & 3 & janv-février;avril à juillet \\
H. bimaculatus & 1 & juin-juillet \\
\hline
\end{tabular}

$\mathrm{N}=$ effectif total des individus d'une espèce dans chaque site.

\section{DISCUSSION}

Les variables environnementales ont présenté des variations en fonction des sites et des saisons. Les variations de température $\left(27,9^{\circ} \mathrm{C}-29,6^{\circ} \mathrm{C}\right)$ sont faibles et peuvent se justifier par la densité de la végétation dense du Parc National de Taï sur les rives du lac, et qui joue un rôle important dans la régulation de la température (Pramova et al., 2012). Concernant le taux d'oxygène dissous, les résultats obtenus indiquent de bonnes conditions d'oxygénation du milieu et peuvent traduire un faible degré de minéralisation bactérienne de ces eaux et de des conditions favorables de survie des organismes aquatiques. En effet, selon IGBE (2005), une teneur de 4 à $6 \mathrm{mg} / \mathrm{l}$ caractérise des conditions optimales pour la vie aquatique. La conductivité présente des valeurs généralement plus élevées en saisons des pluies qui seraient liées à l'apport d'eaux contaminées en ions provenant des activités humaines dans les parties situés en amont du fleuve Sassandra comme l'a indiqué Kouamé (2010). Contrairement aux autres paramètres, la transparence et la profondeur des sites ont présenté des variations saisonnières significatives qui seraient liées au débit du fleuve Sassandra, à la pluviométrie et à la fréquence d'ouverture des vannes du barrage. Ces paramètres sont proches des résultats de Goli Bi et al. (2019) et traduisent des conditions optimales de survie pour les larves et adultes de poissons tropicaux (Bénech et Lek, 1981).

La richesse spécifique (32 espèces) obtenue dans la présente étude est dominée par la famille des Cichlidae $(22 \%)$. Cette dominance par rapport aux autres familles peut être justifiée par leur capacité à supporter une gamme variée de conditions environnementales (Paugy et al., 2006). Toutefois, le nombre d'espèces est relativement faible en comparaison aux travaux de Goli $\mathrm{Bi}$ et al. (2019) qui ont signalé la présence de 45 espèces. En effet, ces auteurs ont échantillonné à Buyo ville et à Guessabo, à l'aide de plusieurs types d'engins (filets maillants, nasse) alors que les échantillonnages dans la présente étude se sont limités à une portion du lac au sein du Parc National de Taï. Ces différences seraient alors liées à la surface d'eau échantillonnée qui a été restreinte dans ce travail et aux techniques de capture des poissons. Sur la base de l'occurrence et de l'abondance des stades sexuels IV, V et VI, il apparait que les géniteurs de 14 espèces fraient dans tous les sites qui ont fait l'objet d'échantillonnage. Parmi elles, Barbus macrops et C. zillii qui reproduisent dans la majorité des sites échantillonnés. Cela peut s'expliquer par le comportement de ponte de ces espèces. En effet, les genres Coptodon et Sarotherodon ont des pontes multiples et pondent pratiquement toute l'année (Lowe-Mcconnell, 1987). Ce mode de reproduction peut justifier la fréquentation de plusieurs sites de frai. En effet, les géniteurs de $C$. zillii ont été capturés durant 8 campagnes de pêche dans le lac de Buyo. Par contre, des individus matures de plusieurs espèces dont Mormyrops anguilloides, Distichodus rostratus, Labeo coubie, L. parvus, L. senegalensis n'ont pas été observés dans les sites prospectés. Cela serait lié à l'utilisation d'autres sites pour la reproduction ou le faible nombre d'échantillons récoltés pour ces espèces. 
D'ailleurs, il est probable que les sites échantillonnés représentent aussi des frayères pour les espèces dont le nombre d'individus récoltés a été faible $(\mathrm{N}<10)$. Par ailleurs, Balon (1990) a distingué 3 modes de reproduction chez les poissons: les pondeurs pélagiques qui ne s'occupent pas des œufs pondus, les poissons qui gardent leurs œufs soit dans des nids, soit dans des substrats préalablement aménagés et ceux qui transportent leurs œufs durant au moins une partie de la période embryonnaire. Ces espèces pourraient être des pondeurs pélagiques n'ayant pas besoin de sites spécifiques de frai.

Les périodes de capture d'individus en phase de reproduction peuvent donner des indications sur les saisons de reproduction des poissons. D'après LoweMcconnell (1987), 3 types de comportements reproducteurs peuvent être évoqués chez les poissons

: (1) les espèces à une seule ou courte saison de reproduction (ponte unique), (2) celles dont la saison de reproduction est plus étalée dans l'année (ponte fractionnée) et (3) celles qui pondent pratiquement toute l'année (ponte multiples). Ainsi, les six espèces suivantes Heterotis niloticus, Brycinus longipinnis, Chrysichthys maurus, Clarias anguillaris, Malapterurus electricus et Hemichromis bimaculatus ayant été capturés seulement durant une ou deux campagnes consécutives (juin-juillet et septembre-octobre) (Tableau 5), peuvent être considérées comme ayant une saison unique de reproduction tandis que les autres espèces ont une saison de reproduction plus étalée. Parmi les espèces à reproduction étalée, les femelles matures de $S$. koensis ont été capturées durant 4 campagnes d'échantillonnage entre juillet et octobre. Nos résultats sont comparables à ceux de Kouassi et al. (2019) qui ont étudié les caractéristiques de reproduction de $S$. koensis dans le lac de Buyo et qui ont indiqué que cette espèce s'y reproduisait entre juillet et novembre avec des pics de reproduction en août et octobre. Par ailleurs, la plupart des géniteurs en phase de reproduction a été capturé entre juin et juillet. Cela peut se justifier par la crue à cette période où il y a une augmentation de la profondeur consécutive à plus un grand nombre d'habitats et de la disponibilité en

\section{CONCLUSION}

Cette étude a permis de recenser 32 espèces de poisson dont les géniteurs de 14 espèces fréquentent, pour leur reproduction, les sites d'échantillonnage retenus dans la partie du lac de Buyo située dans le Parc National de Taï (PNT). Si certaines espèces ressources alimentaires pour de nombreuses espèces (Castillo-Rivera, 2013). Concernant les sites de frai, les résultats ont montré que les espèces Chrysichthys maurus (S4), Malapterurus electricus (S6), Synodontis punctifer (S3) et Hemichromis bimaculatus (S6) sont spécifiques à un seul site de frai. En effet, les sédiments des sites S3 et S4 sont dominés par du gravier tandis que les sédiments de S6 sont sableux. Cette ségrégation des sites de frai pourrait être liée aux différences de choix des micro-habitats de fraie et le comportement reproducteur des poissons et serviraient, dans certains cas, à réduire la compétition entre ces espèces (Comptois et al., 2004). Le choix d'un seul site peut également témoigner d'un comportement territorial de l'espèce qui consiste à la défense d'un espace contre la prédation et à la construction de nids pour les soins parentaux (Mckaye et al., 1994). En effet, Parkinson et al. (1999) ont montré que les poissons lithophiles pondeurs sur gravier modifient activement le milieu de vie de leur progéniture, ce qui tend à maximiser le succès de la reproduction. Par ailleurs, d'autres travaux ont montré que pour les espèces qui construisent des nids, la morphologie des nids ainsi que la nature des sédiments utilisés pour leur construction pouvaient varier d'une espèce de poissons à l'autre. Cette variété de nids est bien connue chez des Cichlidae où les œufs sont déposés dans des cuvettes aménagées sur le sable ou la vase et où ils sont fertilisés pour éclore quelques jours après durant lesquels les deux parents assurent en général une garde vigilante (Fryer et lles, 1972). Chez d'autres espèces telles que Heterotis niloticus, les nids sont des dépressions aménagées dans le sédiment alors qu'ils se présentent sous forme de terriers chez Protopterus annectens (Fermon et Bigorne, 2006). Les résultats de la présente étude montrent ainsi que si des espèces comme $B$. macrops et $C$. coptodon fraient sur une plus large gamme de sites (surtout ceux dont le substrat est dominé par du sable), par contre, fraient sur un nombre réduit de sites, comme c'est le cas de Chrysichthys maurus et Clarias anguillaris qui semblent préférer, pour leur reproduction, des sites dont le sédiment est dominé par du gravier (S4).

semblent, pour leur reproduction, accepter une plus large gamme de substrats dominé par du sable, d'autres, en revanche, ne se reproduisent que sur un nombre réduit de sites. 


\section{REMERCIEMENTS}

Le présent travail se situe dans le cadre du projet intitulé "Etude des frayères de l'ichtyofaune du lac de Buyo dans le Parc National de Taï au sud-ouest de la Côte d'Ivoire : identification et caractérisation". Les

\section{REFERENCES BIBLIOGRAPHIQUES}

Balon EK, 1990. Epigenesis of an epigeneticist: the development of some alternative concepts on the early ontogeny and evolution of fishes. Guelph ichthyology reviews 1: 1-42.

Bandara NSKV. and Amarasinghe US, 2017. Factors Related to Nesting Sites of Oreochromis niloticus (Linnaeus 1758; Cichlidae) in Irrigation Reservoirs, Sri Lanka. Asian fisheries science 30: 319-335.

Bénech L. and Lek S, 1981. Résistance à l'hypoxie et observations écologiques pour seize espèces de poissons du Tchad. Revue d'Hydrobiologie tropicale 14 (2): 153-108.

Bruton MN. and Gophen M, 1992. The effect of environmental factors on the nesting and courtship behaviour of Tilapia zillii in Lake Kinneret (Israel). Hydrobiologia 239: 171-178.

Comptois A, Chapleau F, Renaud CB, Fournier H, Campbelle B, Pariseau R, 2004. Inventaire printannier d'une frayère multispécifique : l'ichtyofaune des rapides de la rivière Gatineau, Québec. The Canadian FieldNaturalist, 118: 522-529.

Daily GC, Söderqvist T, Aniyar S, 2000. The value of nature and the nature of value. Science 289: 395-396.

Fontana A, 1969. Étude de la maturité sexuelle des sardinelles Sardinella ebo (val) et Sardinelle aurita $\mathrm{C}$. de la région de Pointe Noire. Cahiers ORSTOM, série Océanographie 2(2): 101-114.

Fermon Y. and Bigorne R, 2006. L'Ethologie. In : Les poissons des eaux continentales africaines: Diversité, écologie, utilisation par l'homme (Lévêque C. and Paugy D, eds). IRD Editions, Paris, 225-252 pp.

Fryer G. and Iles TD, 1972. The cichlid fishes of the Great Lakes of Africa: their biology and evolution. Edinburgh, Tropical Fish Hobbyist Publ., London, Oliver and Boyd, 641 pp.

Goli Bi BEP, Kamelan TM, Kien KB, Berté S, Kouamélan EP, 2019. The spatio-temporal dynamics of the fish assemblage of the manmade Lake Buyo (Côte d'Ivoire, West Africa). auteurs remercient le Programme d'Appui Stratégique pour la Recherche et l'Enseignement Supérieur (PASRES) et l'Office Ivoirien des Parcs et Réserves (OIPR) pour leurs appuis financiers.

International Journal of Fisheries and Aquaculture 11(3): 72-85.

Heyman WD. and Kjerfve B, 2008. Characterization of transient multi-species reef fish spawning aggregations at Gladden Spit, Belize. Bulletin of marine science 83(3): 531-551.

IBGE, 2005. Qualité physico-chimique et chimique des eaux de surface : cadre général. Institut Bruxellois pour la Gestion de l'Environnement. Observatoire des Données de l'Environnement, 16 pp.

Kouamé MK, Ouattara A, Dietoa MY, Gourène G, 2006. Alimentation du Clupeidae Pellonula leonensis dans le lac de barrage de Buyo (Côte d'Ivoire). Cybium 30(2): 145-150.

Kouassi KHD, Kamelan TM, Berté S, Kouamélan EP, 2019. Paramètres de reproduction de Synodontis koensis Pellegrin, 1933 (Siluriformes, Mochokidae) dans le lac de barrage de Buyo (Côte d'Ivoire). Journal of Applied Biosciences 132: 13461-13474.

Lowe-Mcconnell RH, 1987. Ecological studies in tropical fish communities. Cambridge, Cambridge University Press, Tropical Biology series, $382 \mathrm{pp}$.

Mckaye KR, Mughogho DE, Stauffer JR, 1994. Sex-role differentiation in feeding and defence of young by a parental catfish, Bagrus meridionalis. Animal behaviour 48: 587-596.

N'Goran YN, 1995. Biologie, écologie et pêche de l'ethmalose : Ethmalosa Fimbriata (Bowdich, 1825) en lagune Aby (Côte d'Ivoire). Thèse de Doctorat, Université de Bretagne Occidentale, $195 \mathrm{pp}$.

Ostendorp W, Dienst M, Jacoby H, Kramer I, Peintinger M, Schmieder K, Werner S, 2004. General framework for a professional evaluation system for lakeshore conservation and water body protection, using Lake Constance as an Example. Expertise of the Arbeitsgruppe Bodenseeufer (AGBU) for the BodenseeStiftung and the Global Nature Fund, Radolfzell, 24pp. 
Parkinson D, Petit F, Perpinien G, Philippart JC, 1999. Habitats de reproduction des poissons et processus géomorphologiques dans des rivières à fond caillouteux. Essai de synthèse et applications à quelques rivières du bassin de la Meuse. Bulletin de la Société géographique de Liège $36: 31-52$.

Paugy D, Lévêque C, Duponchelle F, 2006. La reproduction. In: Les poissons des eaux continentales africaines: Diversité, écologie, utilisation par l'homme (Lévêque $\mathrm{C}$. and Paugy $\mathrm{D}$, eds). IRD Editions, Paris, 147-176 pp.

Paugy D, Lévêque C, Teugels GG, 2003a. Poissons d'eaux douces et saumâtres de l'Afrique de I'Ouest. Tome I. Editions IRD, Paris, Tervuren, $457 \mathrm{pp}$.

Paugy D, Lévêque C, Teugels GG, 2003b. Poissons d'eaux douces et saumâtres de l'Afrique de l'Ouest. Tome II. Editions IRD, Paris, Tervuren, $815 \mathrm{pp}$.

Pimm SL, Russell GJ, Gittlemann JL and Brooks TM, 1995. The future of biodiversity. Science 269 : 347-350.

Pramova E, Locatelli B, Djoudi H, Somorin O, 2012. Forests and trees for social adaptation to climate variability and change. WIREs Climate Change 3: 581-596.

Rosenzweig ML, 2003. Reconciliation ecology and the future of species diversity. Oryx, 37(2): 194205.

Sanderson EW, Jaiteh M, Levy MA, Redford KH, Wannebo AV, Woolmer G, 2002. The human, footprint and the last of the wild. BioScience 52: 891-904.

Traoré K, 1996. État des connaissances sur les pêcheries continentales ivoiriennes. Rapport de consultation, Projet F.A.O. TCP, 135 pp. 